\title{
Transverse emittance growth caused by space-charge-induced resonance
}

\author{
Takaaki Yasuiø* \\ Department of Physics, The University of Tokyo, Tokyo 113-0033, Japan \\ Susumu Igarashi, Yoichi Sato, Tadashi Koseki, ${ }^{\dagger}$ and Kazuhito Ohmi \\ KEK, Tsukuba, Ibaraki 305-0801, Japan
}

(Received 9 September 2019; accepted 1 June 2020; published 16 June 2020)

\begin{abstract}
Transverse emittance growth due to space-charge-induced resonance was studied for high-power beam operation in the J-PARC main ring synchrotron. The turn-by-turn beam sizes were measured near the structure resonance at $\nu_{x}=21$ and near the nonstructure resonance at $\nu_{x}=22$. A clear difference in the emittances was observed. The emittance was observed to grow by 5 times at the operating tune $\nu_{x}=21.10$, and by 1.8 times at $\nu_{x}=22.05$ in the first 100 turns. The results show the advantage of tuning in the region of $\nu_{x}=22$ for high intensity beam operation. The turn-by-turn emittance varied depending on the horizontal tune in the region of $\nu_{x}=21$. This indicates that the mechanism of emittance growth is related to the tune spread distribution. The results were satisfactorily reproduced by the simulations of the beam dynamics, including the space-charge effect incorporated via the particle-in-cell algorithm. To reveal the mechanism of the emittance growth, the space-charge effects on the resonances were evaluated by simulations as well as analytical calculations.
\end{abstract}

DOI: 10.1103/PhysRevAccelBeams.23.061001

\section{INTRODUCTION}

The main ring (MR) of the Japan Proton Accelerator Research Complex (J-PARC)[1] is an intensity-frontier proton accelerator. Protons are injected into the MR with a kinetic energy of $3 \mathrm{GeV}$ and an intensity of $3.3 \times 10^{13}$ protons per bunch (ppb). After injection of 8 bunches, the beam is accelerated to $30 \mathrm{GeV}$ and extracted with an intensity of $2.6 \times 10^{14}$ protons per pulse ( $\mathrm{ppp}$ ) in operation for neutrino experiment. A maximum beam power of $500 \mathrm{~kW}$ has been achieved [2] and we aim to achieve a beam power of $1.3 \mathrm{MW}$ for neutrino operations in the future [3]. It is planned to upgrade the hardware, such as the main magnet power supplies and the rf system and increase the beam intensity to $3.3 \times 10^{14} \mathrm{ppp}$ for the accelerator operation with 1.3-MW beam power [3].

It is crucial to reduce the beam losses to increase the beam intensity. In the recent operation, the beam loss can be localized satisfactorily by collimators. No beam loss is observed due to spilling from the rf bucket. The transverse instability is well suppressed by the feedback system.

\footnotetext{
*tyasui@post.j-parc.jp

Also at Department of Physics, The University of Tokyo, Tokyo, Japan.

Published by the American Physical Society under the terms of the Creative Commons Attribution 4.0 International license. Further distribution of this work must maintain attribution to the author(s) and the published article's title, journal citation, and DOI.
}

The beam losses have been about $1 \%$. Most of them are observed in the period where the energy is below $5 \mathrm{GeV}$, because the space-charge effect is relatively strong. Suppressing the emittance growth is an effective way of reducing beam loss. The emittance growth is mainly caused by some on-resonance particles due to the tune spread. The tune spread appears by the space-charge effect and by the effect of momentum spread of the beam. The typical momentum spread $\Delta p / p$ is 0.004 and the typical chromaticity is $\xi_{x}, \xi_{y}=-7$ in the MR. The chromatic tune spread is estimated to be about 0.03 . On the other hand, depending on the beam intensity, the space-charge tune spread is about 0.2-0.4. The space-charge effect is the predominant factor causing the tune spread in the MR at J-PARC.

To estimate the tune spread distribution [4] in the MR, particle-in-cell simulations, which incorporate the spacecharge effect, have been performed. Figure 1 shows an example of the simulations with the conditions for neutrino operation.

Other than its effect on the tune spread, the space-charge force induces resonances [5-7] that can cause emittance growth when some particles cross it. The space-charge effect induces fourth- or higher-order resonances. The MR has a three-fold symmetry and the space-charge-induced structure resonances in it are $4 \nu_{x}=84,4 \nu_{y}=84$, and $2 \nu_{x}-2 \nu_{y}=0$, as shown in Fig. 1 .

Other resonances are also shown in Fig. 1. The sextupole magnets induce third-order structure resonances at $3 \nu_{x}=63,3 \nu_{y}=63$, and $\nu_{x}-2 \nu_{y}=-21$. The nonstructure 


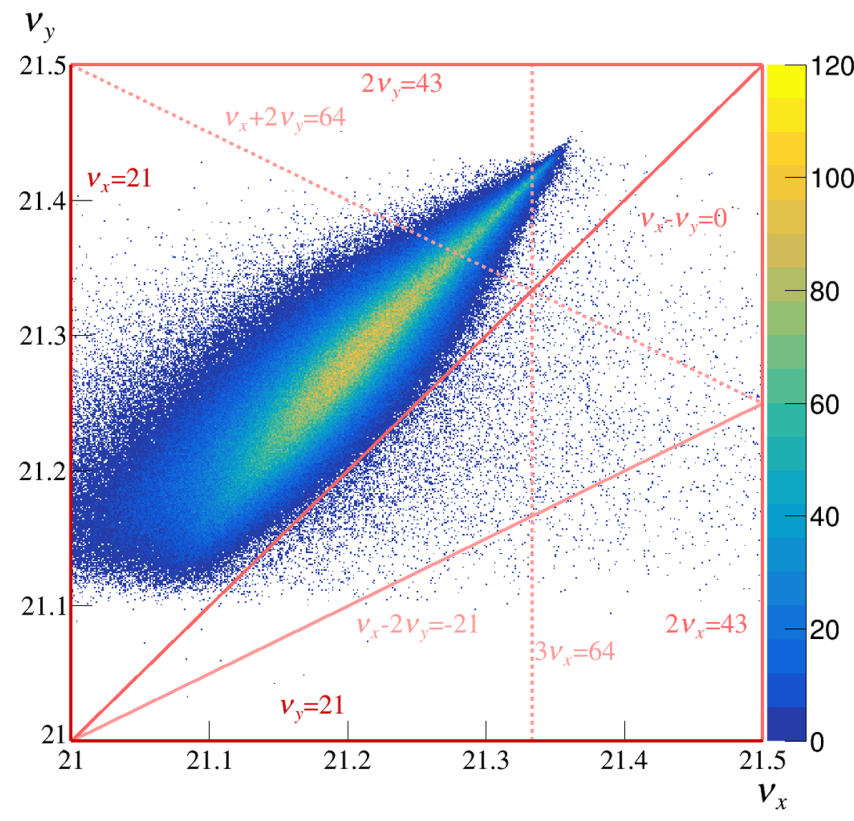

FIG. 1. Simulation of the tune spread and the resonance lines which can cause beam loss. The density of the tune spread distribution is expressed by colors. The simulation was conducted with the following initial conditions: operation point $\left(\nu_{x}, \nu_{y}\right)=$ $(21.35,21.43)$, beam intensity $3.3 \times 10^{13} \mathrm{ppb}$, horizontal emittance $\varepsilon_{x}=5.0 \pi \mathrm{mm} \mathrm{mrad}$, vertical emittance $\varepsilon_{y}=$ $5.5 \pi \mathrm{mm} \mathrm{mrad}$, and bunching factor $B_{f}=0.3$. The initial horizontal distribution was set to a Gaussian, while the initial vertical distribution was set to a parabola which is a typical distribution observed in the MR.

resonances appear at $\nu_{x}+2 \nu_{y}=64$ and $3 \nu_{x}=64$. These resonances are compensated with trim coils of sextupole magnets to reduce the beam loss [2].

In this study, the effects of the space-charge-induced structure resonances at $\nu_{x}=21, \nu_{y}=21$, and the space-charge-induced nonstructure resonance at $\nu_{x}=22$ were evaluated by observing the emittance growth, and the details of this investigation are provided in Sec. II. The difference between the emittance growth due to the resonance at $\nu_{x}=21$ and that at $\nu_{x}=22$ could be clearly observed. The results were reproduced satisfactorily by the space-charge simulations. In Sec. III, the resonance strength was evaluated by Poincaré maps. The resonance strength was evaluated both by simulations and analytical calculations.

\section{EMITTANCE GROWTH MEASUREMENT}

\section{A. Multiribbon profile monitor}

The multiribbon profile monitor (MRPM) [8] situated in the MR was used for measuring the beam profiles. The InjMRPM, located at the beam injection position of the MR, can measure the circulating beam profile in the MR at every turn. Inj-MRPM is in a dispersion-free area. It was confirmed by measurements that there was no significant
TABLE I. Inj-MRPM specifications. The thickness of the electrodes is the same as the ribbons.

\begin{tabular}{lcc}
\hline \hline & Horizontal & Vertical \\
\hline Material & Graphite & Titanium \\
Ribbon pitch & $4.5 \mathrm{~mm}$ & $2.5 \mathrm{~mm}$ \\
Ribbon width & $3.0 \mathrm{~mm}$ & $1.5 \mathrm{~mm}$ \\
Thickness & $3 \mu \mathrm{m}$ & $1 \mu \mathrm{m}$ \\
\hline \hline
\end{tabular}

residual dispersion at the current tune $\left(\nu_{x}, \nu_{y}\right)=$ $(21.35,21.43)$ and at $\left(\nu_{x}, \nu_{y}\right)=(22.30,22.40)$.

Inj-MRPM is made up of five layers, namely in order, solid graphite electrode, graphite ribbons, solid titanium electrode, titanium ribbons, and solid graphite electrode. When the beam passes through the ribbons, secondary electrons are emitted from their surface. The projected beam profile can be obtained by measuring this secondary electron current. On the other hand, beams are deflected by Coulomb scattering at the Inj-MRPM and the scattering angle of the beam particles follows a Gaussian distribution [9]. As the injection beam profile in the transverse direction in the MR is Gaussian, the scattered beam profile is also Gaussian. By convolution calculations, the scattered beam size $\sigma_{n}$ after $n$ turns can be written as

$$
\sigma_{n}^{2}=\sigma_{\text {init }}^{2}+n \sigma_{\text {scat }}^{2},
$$

where $\sigma_{\text {init }}$ represents the initial beam size and $\sigma_{\text {scat }}$ is the growth of the beam size due to multiple scattering after one turn. It is expected that the effect of multiple scattering does not depend on the tune. In this study, 11 ribbons were used in total to measure the horizontal beam profiles. Each ribbon was connected to a readout channel. The specification of the Inj-MRPM is shown in Table I.

\section{B. Principles and Methods}

The experiments were conducted using single bunch beams that were extracted after 100 turns since their injection. Because the synchrotron period is about 600 turns, the effect of the longitudinal motion is small. Three integer resonances $\left(\nu_{x}=21, \nu_{y}=21\right.$, and $\left.\nu_{x}=22\right)$ were the targets of the experiments. For simplicity, we refer to the measurements targeting $\nu_{x}=21$ as the NX21 experiment, $\nu_{y}=21$ as the NY21 experiment, and $\nu_{x}=22$ as the NX22 experiment. Figure 2 shows the operation points and the images of the tune spread in the measurements. For the NX21 experiment, some operation points were chosen in the range of $\left(\nu_{x}, \nu_{y}\right)=(21.25-21.08,21.43)$. The horizontal beam sizes were measured at every turn by the Inj-MRPM for each tune and the horizontal emittance growths were compared for each tune. Similarly, for the NY21 experiment, the operation points were chosen in the range of $\left(\nu_{x}, \nu_{y}\right)=$ $(21.43,21.21-21.07)$ and in the range of $\left(\nu_{x}, \nu_{y}\right)=$ $(22.25-22.05,22.43)$ for the NX22 experiment. Beam loss 


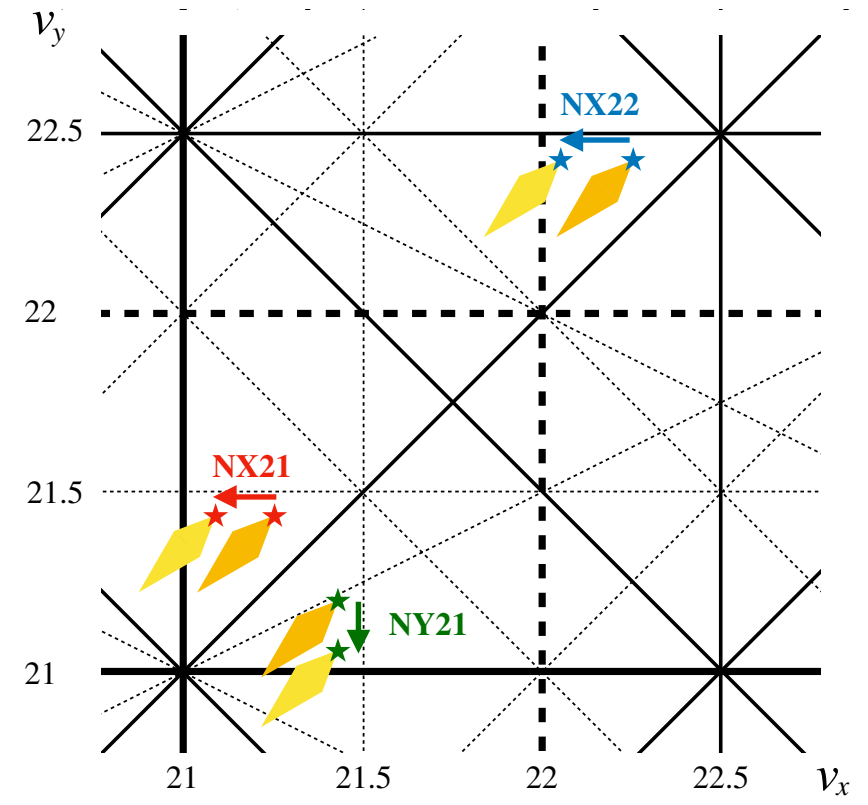

FIG. 2. The operation points set in the experiments (shown as stars), the tune spreads, and the major resonance lines have been plotted. Several operation points were chosen in the range of $\left(\nu_{x}, \nu_{y}\right)=(21.25-21.08,21.43)$ for the NX21 experiment, $(21.43,21.21-21.07)$ for the NY21 experiment, and $(22.25-22.05,22.43)$ for the NX22 experiment. The spacecharge-induced structure resonances have been plotted as solid lines and the other major resonances have been plotted as dotted lines.

was not observed in these ranges. The beam conditions during these experiments are listed in Table II. At these beam intensities, the coherent tune shift caused by the indirect space-charge effect, such as resistive wall impedance, is negligible [10].

The tune spread distribution just after injection keeps its shape even when the tune has changed. If the tune gets closer to an integer resonance line, the number of particles crossing this line will increase. As a result, the emittance grows faster when the number of on-resonance particles is larger. As the MR has a three-fold symmetry, the spacecharge-induced structure resonances appear at $\nu_{x}=21$ and $\nu_{y}=21$. It is expected that the horizontal emittance will grow in the NX21 experiment and the vertical emittance will grow in the NY21 experiments. Meanwhile, $\nu_{x}=22$ is the space-charge-induced nonstructure resonance and is not driven by the space-charge effect unless there are large optics errors. By comparing the emittance growths between the NX21 and NX22 experiments, the difference between the space-charge-induced structure and nonstructure resonances can be evaluated.

The signals of Inj-MRPM were integrated for each channel and each turn to reconstruct the turn-by-turn beam profiles. The beam positions and sizes were calculated for every turn by fitting the integrated signals. The fitting function used was as follows:

$$
f(x)=A\left(1-\frac{1}{n} \frac{\left(x-x_{0}\right)^{2}}{w^{2}}\right)^{n}+C .
$$

This function is identical to a Gaussian for $n \rightarrow \infty$ and to a parabola at $n=1$. The half width at half maximum (HWHM) was taken as the beam size. The tunes were obtained by a discrete Fourier transform of 100 turns of the center positions $x_{0}$. Therefore, the resolution of the tune is estimated within 0.01 .

Though the injected beam is Gaussian, the beam tail grows and the beam profile gets distorted when the tune is close to an integer resonance. This work takes the HWHM as the beam width and focuses on the discussion about the beam core. The cores of the profiles were fitted with good precision by the fitting function given by Eq. (2).

\section{Other contributions to the emittance growth}

The multiple scattering at the Inj-MRPM contributes to a linear growth of the beam emittance. Because there is little optical change at the Inj-MRPM corresponding to different tunes, it can be inferred that the effect of multiple scattering does not depend on the tune. The scattering angle can be estimated from the data in which the tune is far enough from the resonances.

Another cause for the emittance growth is the beam dilution occurring due to the beta mismatch at the injection. The beam size fluctuates during the first several turns and its oscillation is dampened due to the space-charge effect. The amplitude of the oscillation can depend on the tunes because the Twiss parameters at the injection depend on the tunes. To suppress the oscillation, the optics of the transport line was adjusted at each tune.

TABLE II. Conditions of the measurements. The beam intensities were measured by the direct-current current transformers (DCCTs). The natural chromaticity is $\left(\xi_{x}, \xi_{y}\right)=(-28,-29)$.

\begin{tabular}{lccc}
\hline \hline & $\mathrm{NX} 21$ & $\mathrm{NY} 21$ & $\mathrm{NX} 22$ \\
\hline Intensity & $5.3 \times 10^{12} \mathrm{ppb}$ & $3.3 \times 10^{12} \mathrm{ppb}$ & $3.3 \times 10^{12} \mathrm{ppb}$ \\
Tune & $\left(21 .^{*}, 21.43\right)$ & $\left(21.43,21 .^{*}\right)$ & $\left(22 .^{*}, 22.43\right)$ \\
Maximum tune shift & $\left|\Delta \nu_{x}\right|=0.35$ & $\left|\Delta \nu_{x}\right|=0.30$ & $\left|\Delta \nu_{x}\right|=0.30$ \\
& $\left|\Delta \nu_{y}\right|=0.36$ & $\left|\Delta \nu_{y}\right|=0.33$ & $\left|\Delta \nu_{y}\right|=0.33$ \\
Chromaticity & & $\xi_{x}, \xi_{y}=-7$ & \\
\hline \hline
\end{tabular}


Another contribution to the emittance growth is the effect of the sextupoles. In the J-PARC MR, sextupoles are situated in the arc sections. One arc section consists of eight modules. The horizontal phase advance of one module is set at $1.5 \pi \mathrm{rad}$. With this condition, the driving terms of the third-order resonances induced by the sextupoles at $\nu_{x}=21,22$ are canceled. However, the horizontal phase advance of one module is shifted owing to the spacecharge effect. The resonances $\nu_{x}=21,22$, therefore, are excited by the sextupoles even if there are no magnet errors. Details of the evaluation of these effects are provided in Sec. III.

Other than the above-mentioned sextupole effect, the beam encounters unexpected quadrupole fields if there are offsets between the beam position and the center of the sextupoles. Misalignments of the sextupoles and closed orbit errors produce dc offsets and can cause tune shifts and strengthening of resonances. The injection error produces ac offsets. Therefore, the beam size can oscillate with the frequency of the dipole oscillation. The amplitude of oscillation of the quadrupole field produced by the sextupole depends not only on the ac offset, but also on the dc offset and larger when the dc offset is larger. In the experiments, the closed orbit was corrected at $\left(\nu_{x}, \nu_{y}\right)=$ $(21.35,21.43)$ and $(22.30,22.40)$. The correction was not repeated during the tune scan. The closed orbit errors were larger when the tune was closer to the integer resonance. The betatron oscillation amplitude at the beam injection was corrected within $1 \mathrm{~mm}$.

\section{Simulation approach}

\section{Calculation tools}

To obtain the basic optics for the simulations, the magnetic strengths were calculated using the strategic accelerator design (SAD) [11] program. The tracking simulations were performed using the space-charge tracker (SCTR)[4] program. SCTR is a particle-in-cell algorithm code for simulating the beam motion and includes the space-charge effect. The number of macroparticles used in this study was 200,000 .

The calculation of the space-charge forces was conducted as follows. First, the beam distribution was projected onto a transverse mesh. Because the longitudinal beam size is much longer than the transverse beam size in the MR, the transverse space-charge potential was calculated to be satisfied with the two-dimensional Poisson equation. The transverse mesh had the dimensions of $128 \times$ 128 and the mesh size was $0.75 \mathrm{~mm} \times 0.75 \mathrm{~mm}$. Following this, the longitudinal distribution was assigned to the mesh, and the local line density was calculated. The longitudinal mesh number was 128 and the mesh size was $1 \mathrm{~m}$, which is small enough compared to typical beam size of $50 \mathrm{~m}$. Transverse and longitudinal kicks were given by the potential and the local line densities. The space-charge calculation was conducted about every two meters in the MR lattice.

Basically, the space-charge effect on the beam was recalculated at every turn by recomputing the space-charge potential. However, sometimes it is effective to fix the space-charge potential. We call this the "frozen model." In the frozen model, beams experience the same space-chargedriven multipolar field effect at every turn. It is useful to discuss the space-charge-induced resonance based on the initial beam distribution.

\section{Simulation conditions}

Simulations were performed using the same initial conditions as the experiments and the details of the conditions are given in Table III.

The optics parameters corresponding to each tune were calculated by SAD. They included the magnets alignment errors and strength errors [12]. These parameters were transferred to SCTR for particle tracking.

The initial transverse emittance was determined by the shots whose tunes were far from the integer resonance. Figure 3 shows the averaged beam sizes of the five shots whose tunes were around $\left(\nu_{x}, \nu_{y}\right)=(21.25,21.43)$. The beam size fluctuated during the first 10 turns due to the beta mismatch at the injection. The fluctuation damped within 10 turns due to dilution. In order to evaluate the injection beam size and the scattering angle, the data from turn 11 to turn 100 were fitted to a linear function [Eq. (1)]. The initial beam size was determined as $\sigma_{\text {init }}$ of the function [Eq. (1)]. The beta function at Inj-MRPM of $\nu_{x}=21.25$ is $16.3 \mathrm{~m}$ as

TABLE III. Initial conditions of the simulations.

\begin{tabular}{lccc}
\hline \hline & $\mathrm{NX} 21$ & $\mathrm{NY} 21$ & $\mathrm{NX} 22$ \\
\hline $\begin{array}{l}\text { Intensity } \\
\text { Tune }\end{array}$ & $5.3 \times 10^{12} \mathrm{ppb}$ & $3.3 \times 10^{12} \mathrm{ppb}$ & $3.3 \times 10^{12} \mathrm{ppb}$ \\
Transverse distribution & $\left(21 .^{*}, 21.43\right)$ & $\left(21.43,21 .^{*}\right)$ & $(22 . *, 22.43)$ \\
Transverse emittance & Gaussian & \\
$\begin{array}{l}\text { Longitudinal distribution } \\
\text { Longitudinal beam size }\end{array}$ & $1.1 \pi \mathrm{mm} \mathrm{mrad}$ & $0.85 \pi \mathrm{mm} \mathrm{mrad}$ & $0.85 \pi \mathrm{mm} \mathrm{mrad}$ \\
$\begin{array}{l}\text { Momentum spread } \\
\text { Chromaticity }\end{array}$ & $52 \pm 1 \mathrm{~m}$ & Parabola & $46 \pm 2 \mathrm{~m}$ \\
\hline \hline
\end{tabular}




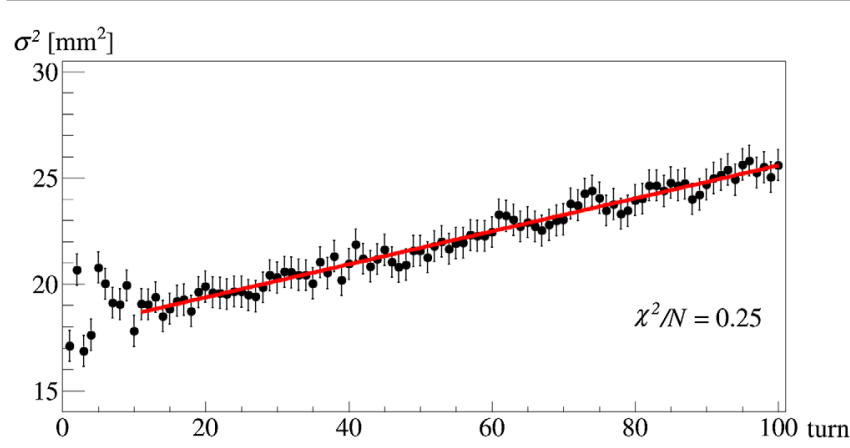

FIG. 3. Average of the beam sizes of five shots for each turn shown as plots. The error bars are the RMS of the five shots. The first 10 data points show fluctuations because of the beta mismatch at the injection. The red line represents the linear fitting to the plots.

per the results obtained from SAD. As Inj-MRPM is located in a dispersion-free area, the emittance was calculated by $\epsilon=\sigma_{\text {init }}^{2} / \beta$. The same procedure was carried out for the NY21 and NX22 experiments. The initial beam distribution was prepared to reproduce the effect of the residual beta mismatch at the injection.

The longitudinal beam profiles were measured by the wall current monitor [13]. The longitudinal distribution was observed to be parabolic. We define the bunching factor, $B_{f}$, as the ratio of the average amplitude to the peak amplitude in the wall current monitor, and for the beam profile, $B_{f} \sim 0.2$. The momentum spreads were adjusted so that the longitudinal beam profiles at the 100 turns of the simulations were the same as those in the experiments. The injection errors were not applied in the simulations.

The multiple scattering at Inj-MRPM was implemented in the simulations. Each particle is scattered turn by turn with a random angle following a Gaussian distribution. The root mean square (RMS) of the scattering angles was $\theta_{x, \mathrm{rms}}=24 \mu \mathrm{rad}$. It was determined such that the emittance growth rate of the simulations, where the tunes were far from the integer resonance, matched the experiments. The scattering angle can also be calculated as [9]

$\theta_{x, \mathrm{rms}}=\frac{13.6 \mathrm{MeV}}{\beta_{\text {rel }} c p} z \sqrt{\frac{x}{X_{0}}}\left[1+0.038 \log \left(\frac{x z^{2}}{X_{0} \beta_{\mathrm{rel}}^{2}}\right)\right]$,

where $\beta_{\text {rel }} c, p$, and $z$ are the velocity, momentum, and charge number of the beam particle respectively, $x$ is the thickness of the target, and $X_{0}$ is the radiation length of the target. Using this expression, the RMS of the scattering angle at each layer was calculated. The thicknesses of targets are given in Table I and the radiation lengths are $42.70 \mathrm{~g} / \mathrm{cm}^{2}$ (Graphite) and $16.17 \mathrm{~g} / \mathrm{cm}^{2}$ (Titanium) [14]. The total scattering angle, derived by square root of the sum of squares of each angle, was $\theta_{x, \mathrm{rms}}=20 \mu \mathrm{rad}$ and is consistent with the simulation results.
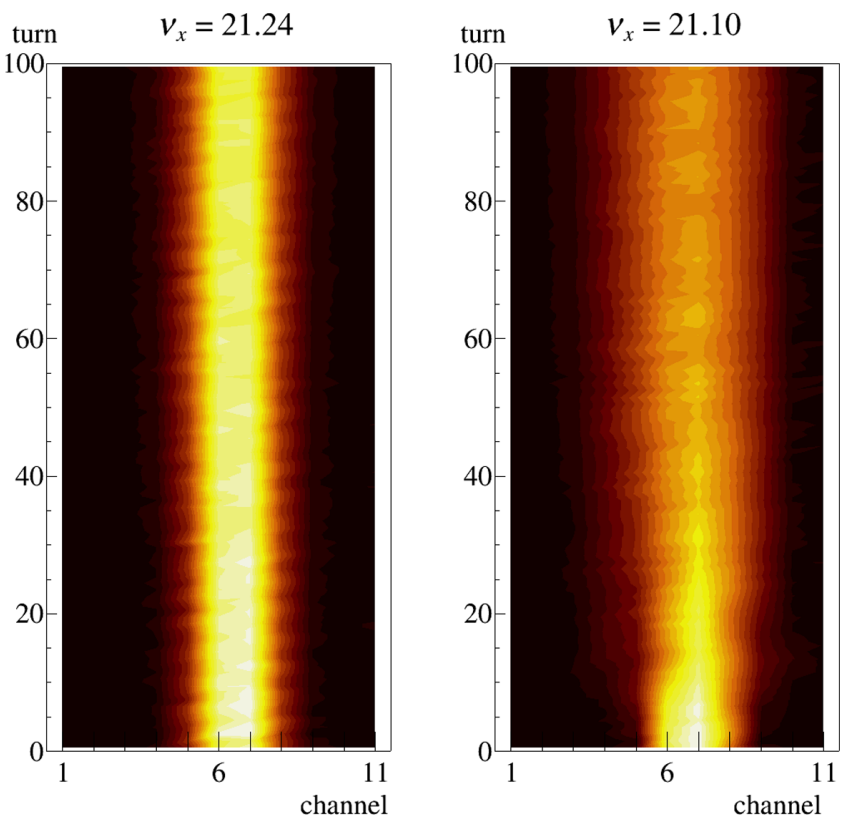

FIG. 4. Integrated signals of Inj-MRPM in the horizontal plane as a function of turns. The figure on the left shows the result corresponding to the condition of $\left(\nu_{x}, \nu_{y}\right)=(21.24,21.43)$, and the one on the right shows that corresponding to the condition of $\left(\nu_{x}, \nu_{y}\right)=(21.10,21.43)$. The horizontal axes represent the channels and the vertical axes represent the turns. The pitch of the adjacent channels was $4.5 \mathrm{~mm}$.

\section{E. Results}

Figure 4 shows examples of the integrated signals of the Inj-MRPM in the horizontal plane. The picture on the left is the result of $\left(\nu_{x}, \nu_{y}\right)=(21.24,21.43)$. No clear dipole or quadrupole oscillations were seen. The picture on the right is the result of $\left(\nu_{x}, \nu_{y}\right)=(21.10,21.43)$. The emittance grew rapidly in 100 turns. After about 40 turns, the beam size oscillated over a 10-turn period. This corresponds to the frequency of the dipole oscillation and is interpreted as the effect of the beam orbit distortions.

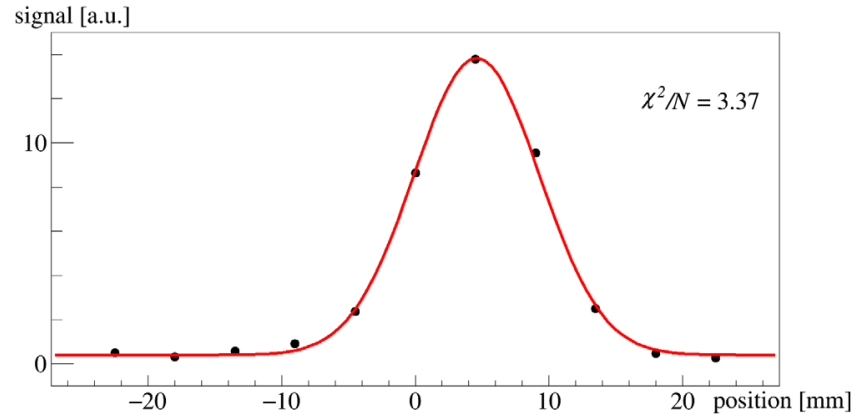

FIG. 5. Example of integrated signals of Inj-MRPM. The black points represent the integrated signals of Inj-MRPM and the red line represents the fitting. This is the data at the turn 10 of the beam and corresponding to the condition $\left(\nu_{x}, \nu_{y}\right)=(21.08,21.43)$. 

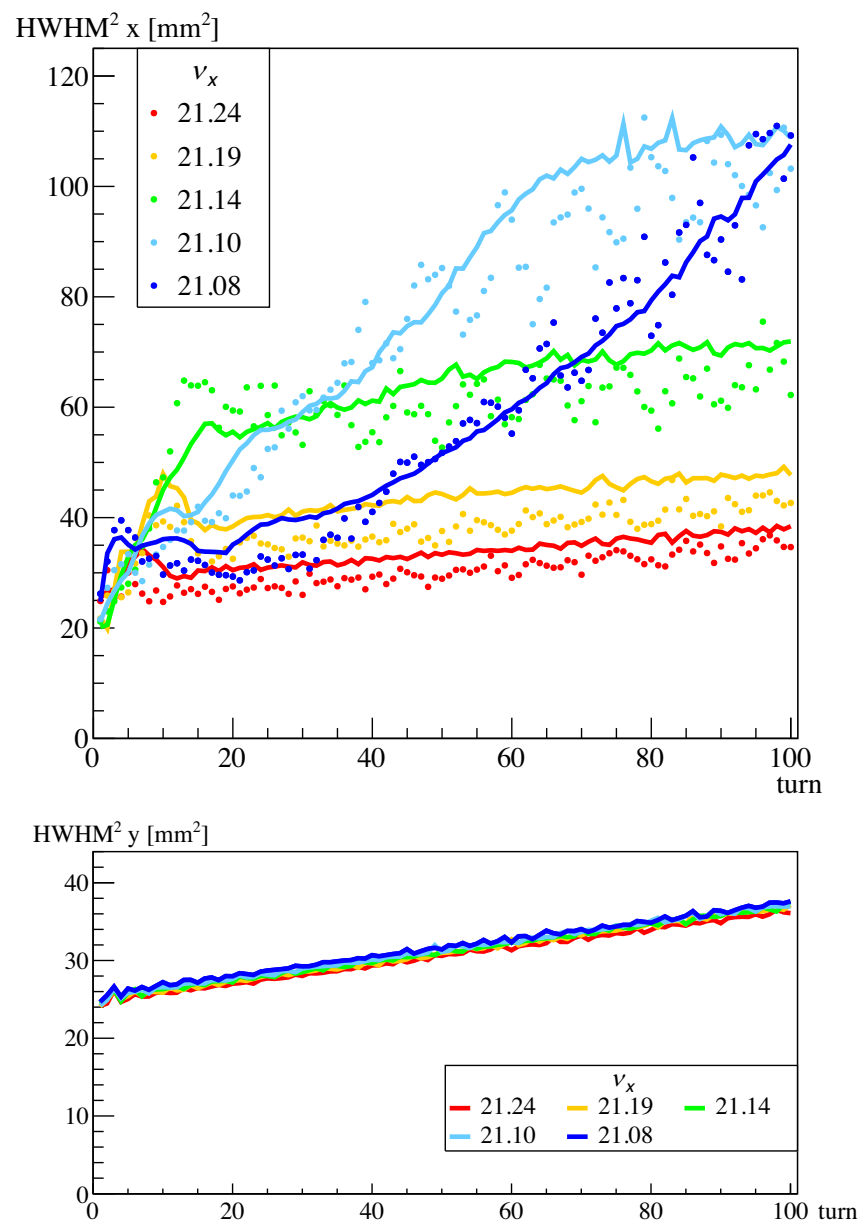

FIG. 6. Beam sizes in the NX21 experiment. The upper picture shows the horizontal beam sizes and the lower picture shows the vertical ones. Experimental results are shown as circles and simulation results are shown as lines. The color difference represents the difference in the horizontal tunes. The vertical tunes were fixed to $\nu_{y}=21.43$.

Figure 5 shows the integrated signals of the Inj-MRPM and a corresponding fitting to the data points. Under the condition that the tune was near the integer resonance, the profile was distorted from a Gaussian.

Figures 6, 7, and 8 show the beam sizes as a function of the number of turns in the NX21, NY21, and NX22 experiments respectively. The results of the measurements are shown as circles, while the simulation results are shown as solid lines. The experimental data were taken only in the horizontal plane.

The simulation results were observed to be in good agreement with the experimental results in all conditions. Though the simulations contained magnetic errors and alignment errors in Figs. 6-8, the results of the NX21 experiment can be reproduced only by the effect of the space-charge and multiple scattering. That is to say that the emittance growth is derived from the space-charge effect in the NX21 experiments. On the other hand, the differences
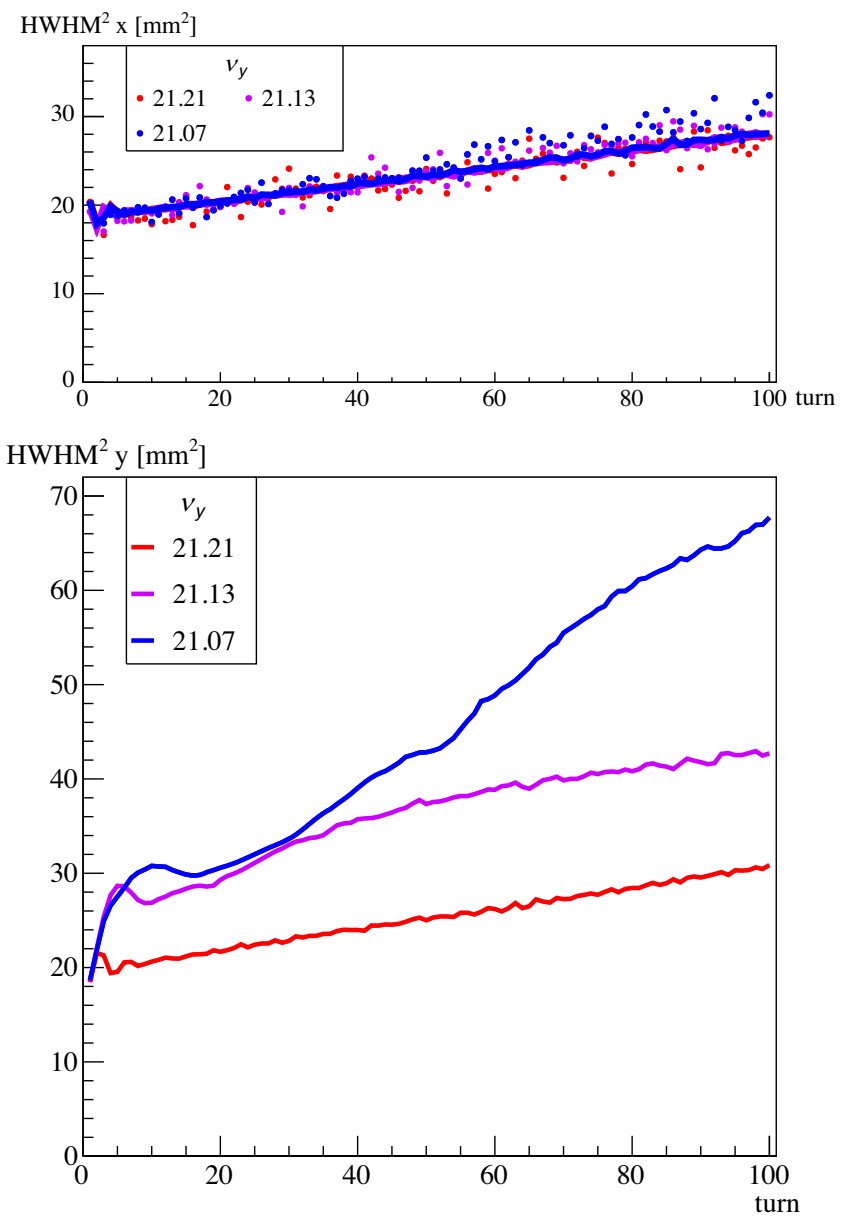

FIG. 7. Beam sizes in the NY21 experiment. The upper picture shows the horizontal beam sizes and the lower picture shows the vertical ones. Experimental results are shown as circles and simulation results are shown as lines. The color difference represents the difference in the vertical tune. The horizontal tunes were fixed to $\nu_{x}=21.43$.

in the emittance growth ratios in the NX22 experiment could be reproduced only by employing errors such as closed orbit errors.

In the NX21 experiment, the turn-by-turn growth of the horizontal beam sizes strongly depended on the horizontal tune. The emittance growth ratio in 100 turns was 1.5 at $\nu_{x}=21.24$ and 5 at $\nu_{x}=21.10$ in the experiments. The growth of the vertical beam sizes did not depend on the horizontal tune in the simulations. In the NY21 experiment, the growth behavior of the horizontal beam sizes did not depend on the vertical tune. The emittance grew by a factor of 1.6 for all tunes in the experiments. The behavior of the vertical beam sizes strongly depended on the vertical tune in the simulations. With these two experiments, it is clear that $\nu_{x}=21$ affected only the horizontal profiles and $\nu_{y}=21$ affected only the vertical profiles. The linear emittance growths, which are not affected by the resonance, are derived from the multiple scattering at the Inj-MRPM. 


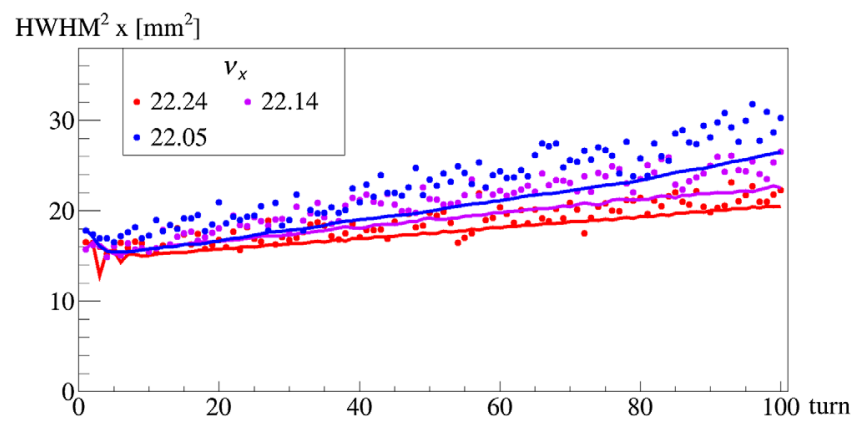

FIG. 8. Horizontal beam sizes in the NX22 experiment. The circles represent the results of the measurements. The color difference represents the difference in the horizontal tunes, which are shown in the upper left part of the figure. The vertical tunes were fixed to $\nu_{y}=22.43$. The lines correspond to the simulation results for each condition.

In the NX22 experiment, the beam size grew almost linearly with the turns. The growth ratio depended weakly on the horizontal tune: 1.4 at $\nu_{x}=22.24$ and 1.8 at $\nu_{x}=$ 22.05 in the experiments. As compared to the NX21 experiment, the beam was observed to grow much slower in the NX22 experiment. Although the tune spread was smaller by a factor of 0.86 in the NX22 experiment, it cannot explain the slow growth. The measured difference between the NX21 and the NX22 experiments is exactly the difference between the structure resonance and the nonstructure resonance. The main reason for the emittance growth in the NX22 experiment is the multiple scattering at Inj-MRPM.

The emittance growth in the NX21 experiment reflected the tune spread distribution of the injected beam. In the case of $\nu_{x}=21.24$, the beam size grew slowly and linearly. This is because the resonance $\nu_{x}=21$ was far enough and multiple scattering made growth. For the case of $\nu_{x}=21.14$, there was a fast growth in the first 15 turns. This can be interpreted as the core of the tune spread distribution crossing $\nu_{x}=21$ at the injection, resulting in a rapid emittance growth. After turn 15, the tune spread was smaller owing to the emittance growth and the major part of the tune spread was above $\nu_{x}=21$. The emittance growth became slow and linear owing to multiple scattering. In the case of $\nu_{x}=21.08$, the emittance was temporarily stable in the first 30 turns. This can be understood to be due to the core of the tune spread being below $\nu_{x}=21$. The integer resonance had little effect on the particles at the beam center. The core of the tune spread gradually moved close to the integer resonance line because the beam tail crossing the integer resonance line grew and the space-charge force was weakened. The core of the tune spread began crossing the integer resonance after turn 30, causing a rapid emittance growth. From these considerations, the core of the tune spread distribution at the injection was thought to be around -0.14 from the operation point.
In some cases, a small systematic difference is observed between the results of the experiments and simulations corresponding to NX21. One of the reasons is the systematic error in the background estimations of the signals in the experiments. There were only 11 channels used for the measurements, and this made it difficult to evaluate the signal background. The small beam sizes in the experiments can be explained by the background overestimation. Another possible cause of the systematic difference is that the initial beam distribution might have deviated from the Gaussian distribution. A real detailed distribution could be obtained if there were more channels.

\section{EVALUATION OF THE RESONANCE STRENGTH}

\section{A. Frozen model simulations}

The increase in the horizontal emittance in the NX21 experiment strongly depended on the horizontal tunes. This is because the number of particles crossing the resonance was changed owing to the tune spread. The main source of the resonance $\nu_{x}=21$ was space-charge. In order to evaluate the effect of the space-charge-induced resonance on particles, frozen model simulations were conducted employing the SCTR code. The frozen model fixes the space-charge potential at the conditions of the first superperiod of the first turn. Because the space-charge forces are fixed, the beam feels the same multipole fields at every superperiod. As a result, space-charge-induced resonances appear clearly. It is effective to discuss the space-chargeinduced resonance at the first superperiod. The effects of multiple scattering and errors of magnets were not included in order to make the discussion simpler.

Figure 9 shows the Poincare maps with the condition of the bare tune $\nu_{x 0}=21.24,21.14$, and 21.08 using the frozen model. The figures in the top row are drawn with the normalized phase-space coordinates $x-p_{x}$. If a particle is far enough from the resonance, it traces a round trajectory in the normalized phase-space coordinates. On the other hand, if it is captured by some resonance, its trajectory will be an island. The figures in the bottom row are drawn with the action-angle coordinates $\psi_{x}-J_{x}$. When a particle is far from resonances, its action is constant. If a particle is near resonances, its action changes turn by turn. The incoherent tunes of the particles drawn in Fig. 9 are shown in Fig. 10.

From the Poincaré maps of $\nu_{x 0}=21.24$, no clear resonance island was observed. This explains the small emittance growth at $\nu_{x 0}=21.24$. From the Poincaré maps of $\nu_{x 0}=21.14$, it can be seen that the particles whose actions were $2 J_{x} \lesssim 7 \mu \mathrm{m}$ were affected by the resonance. From Fig. 10, it is confirmed that the incoherent tunes of the particles on the resonance (shown by orange and red colors) are almost 21. Considering that the emittance of the beam was set to $1.1 \pi \mathrm{mm} \mathrm{mrad}$, most of the particles were 

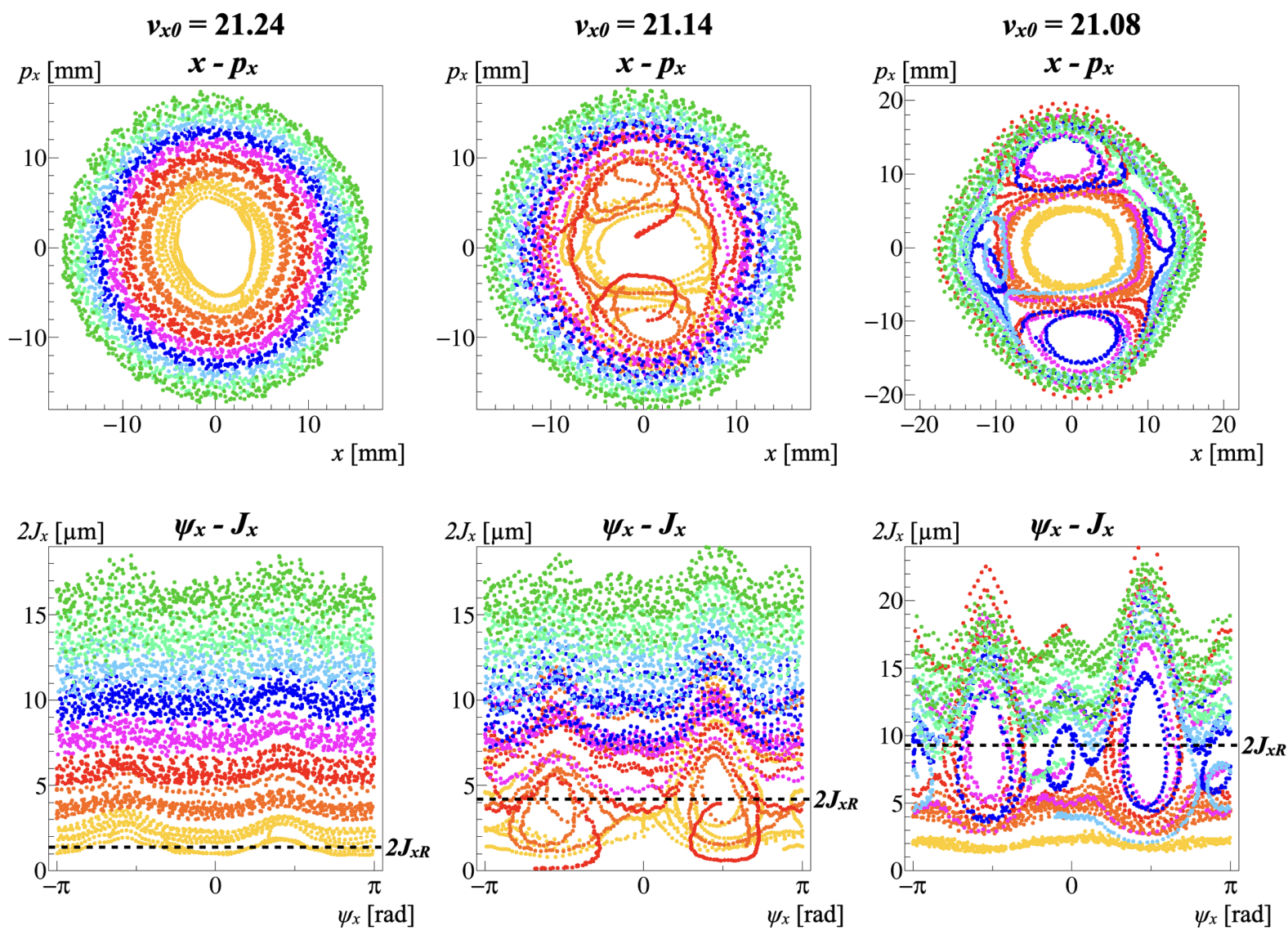

FIG. 9. Poincaré maps obtained from SCTR calculations using the frozen model. The left panel corresponds to $\nu_{x 0}=21.24$, the center panel to $\nu_{x 0}=21.14$, and the right panel to $\nu_{x 0}=21.08$. The top row shows the Poincare maps of the normalized phase-space coordinates and the bottom row shows those of the action-angle coordinates. The same colors represent the same action in all plots. The black dotted lines in the bottom row figures represent the actions which meet the resonance conditions calculated analytically.

under the influence of the space-charge-induced structure resonance.

The Poincaré maps corresponding to $\nu_{x 0}=21.08$ indicate the effect of a fourth-order resonance to be due to the space-charge effect. The particles whose actions were $2 J_{x} \lesssim 4 \mu \mathrm{m}$ were out of the resonance. As can be seen from

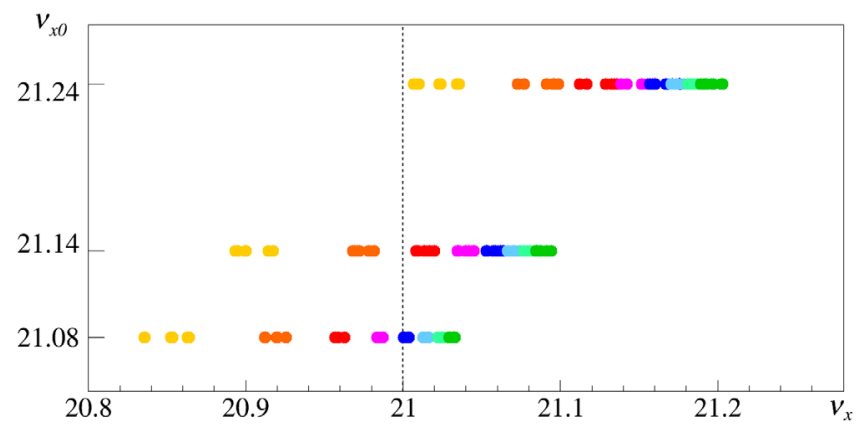

FIG. 10. Incoherent tunes in the NX21 experiment obtained by simulations. The vertical axis represents the bare tune and the horizontal axis represents the incoherent tune. Different actions have been plotted with different colors. The color codes are the same as in Fig. 9.
Fig. 10, the incoherent tune of the orange particles $\left(2 J_{x} \sim 4 \mu \mathrm{m}\right)$ is about 0.08 below 21 , and that of the yellow particles $\left(2 J_{x} \sim 2 \mu \mathrm{m}\right)$ is about 0.15 below 21 . This indicates that the particles around the beam center were out of the range of the space-charge-induced resonance and explains the stable beam size for the first 30 turns.

In the results of $\nu_{x 0}=21.08$, the fourth-order resonance islands are distorted. This is caused by the modulation of the Twiss parameters derived from the space-charge effect. The normalized momentum $p_{x}$ is calculated by using the bare Twiss parameters. The modulation of the Twiss parameters modifies $p_{x}$. Contour lines of the actions in the $x-p_{x}$ coordinates are distorted. In the $x-p_{x}$ coordinates of $\nu_{x 0}=21.08$, the trajectory drawn by the yellow particles $\left(2 J_{x} \sim 2 \mu \mathrm{m}\right)$ looks like an ellipse and indicates modulation of the Twiss parameters.

Figure 11 shows the Poincaré maps of $\nu_{x 0}=22.24,22.14$, and 22.05 obtained using the frozen model simulations. The incoherent tunes in these conditions are shown in Fig. 12. In the case of the Poincare maps of $\nu_{x 0}=22.24$, the beam was not affected by resonances. In fact, the incoherent tunes of the tracking particle were far from 22 , as can been seen from Fig. 12. In the Poincaré maps of $\nu_{x 0}=22.14$, resonance 

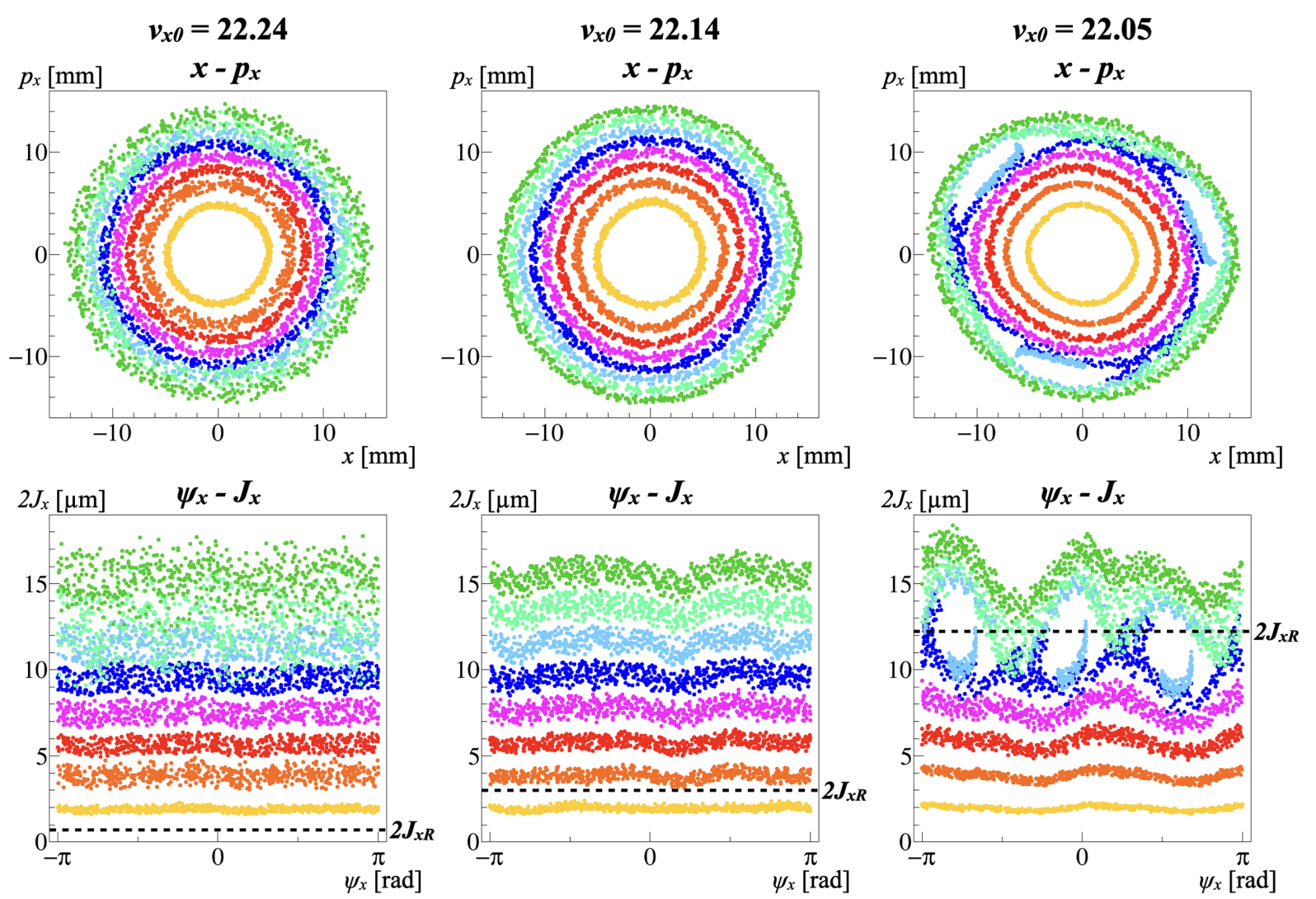

FIG. 11. Poincaré maps obtained from SCTR calculations using the frozen model. The left panel corresponds to $\nu_{x 0}=22.24$, the center panel to $\nu_{x 0}=22.14$, and the right panel to $\nu_{x 0}=22.05$. The top row shows the Poincaré maps of the normalized phase-space coordinates and the bottom row shows those of the action-angle coordinates. The same colors represent the same action in all plots. The black dotted lines in the bottom row represent the actions which meet the resonance conditions calculated analytically.

islands were not observed clearly. Large third-order resonance islands could be seen for the case of $\nu_{x 0}=22.05$. The incoherent tunes of the on-resonance particles were around $\nu_{x}=22$ as is observed from Fig. 12. As the space-charge does not induce odd-order resonances, it is considered that the integer resonance $\nu_{x}=22$ was excited by the sextupoles.

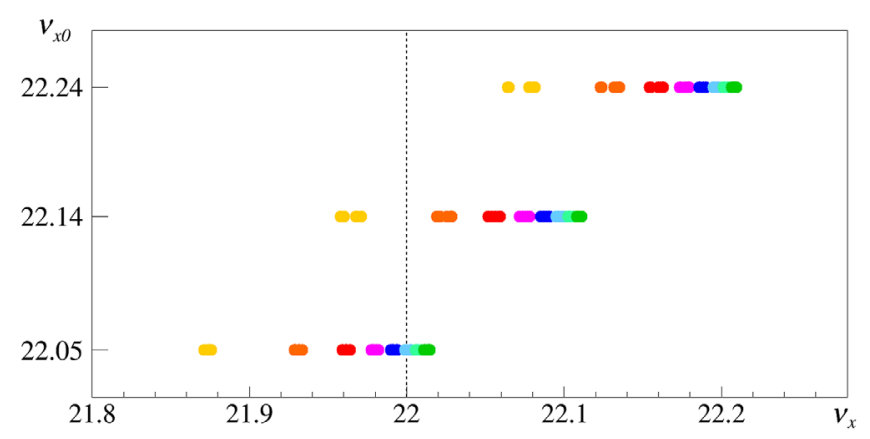

FIG. 12. Incoherent tunes in the NX22 experiment obtained by simulations. The vertical axis represents the bare tune and the horizontal axis represents the incoherent tune. Different actions have been plotted with different colors. The colors are the same as in Fig. 11.
In any tune at $\nu_{x 0}=22 . *$, fourth-order resonance islands did not appear. This is consistent with the fact that $\nu_{x}=22$ is a nonstructure resonance for the space-charge. The integer resonance $\nu_{x}=22$ is not excited by the spacecharge effect unless there are optics errors.

\section{B. Analytical calculations}

\section{Principles}

The resonance islands drawn in Figs. 9 and 11 can be estimated by analytical calculations.

The tunes of the particles in a Gaussian distribution depend on their actions. The space-charge potential of a Gaussian distribution can be written as [15]

$$
U(x, y ; s)=\frac{\lambda r_{0}}{\gamma_{\mathrm{rel}}^{3} \beta_{\mathrm{rel}}^{2}} \int_{0}^{\infty} \mathrm{d} q \frac{\exp \left(-\frac{x^{2}}{2 \sigma_{x}^{2}+q}-\frac{y^{2}}{2 \sigma_{y}^{2}+q}\right)}{\sqrt{2 \sigma_{x}^{2}+q} \sqrt{2 \sigma_{y}^{2}+q}},
$$

where $x, y$ are the transverse positions of the particle, $\lambda$ is the line density of the beam, $r_{0}$ is the classical radius of the particle, $\beta_{\text {rel }}, \gamma_{\text {rel }}$ are the Lorentz factors, and $\sigma_{x}, \sigma_{y}$ are the RMS beam sizes. The Fourier expansion of the potential along the orbit is given by [16] 
$\tilde{U}_{m_{x}, m_{y}}=\frac{1}{(2 \pi)^{2}} \oint \mathrm{d} \psi_{x} \oint \mathrm{d} \psi_{y} \oint \mathrm{d} s U e^{i m_{x} \psi_{x}+i m_{y} \psi_{y}}$,

where $\psi_{x}, \psi_{y}$ are the betatron phases. $\tilde{U}_{00}$ is the phaseindependent term of the potential. The tune shifts $\Delta \nu_{x}$ are calculated using

$$
\begin{aligned}
2 \pi \Delta \nu_{x}= & \frac{\partial \tilde{U}_{00}}{\partial J_{x}} \\
= & -\frac{\lambda r_{0}}{\gamma_{\text {rel }}^{3} \beta_{\text {rel }}^{2}} \oint \mathrm{d} s \frac{\beta_{x}}{\sigma_{x}^{2}} \int_{0}^{\infty} \frac{e^{-w_{x}-w_{y}} \mathrm{~d} \zeta}{(2+\zeta)^{3 / 2}(2 r+\zeta)^{1 / 2}} \\
& \times\left[I_{0}\left(w_{x}\right)-I_{1}\left(w_{x}\right)\right] I_{0}\left(w_{y}\right),
\end{aligned}
$$

where $J_{x}, J_{y}$ are the actions of the particles, $\beta_{x}, \beta_{y}$ are the betatron functions, and $I_{\nu}(x)$ is the modified Bessel function,

$$
\begin{gathered}
r=\sigma_{y}^{2} / \sigma_{x}^{2}, \\
w_{x}=\frac{J_{x} \beta_{x}}{(2+\zeta) \sigma_{x}^{2}}, \\
w_{y}=\frac{J_{y} \beta_{y} r}{(2 r+\zeta) \sigma_{y}^{2}} .
\end{gathered}
$$

In general, the condition that a particle is in resonance can be written as

$$
\begin{aligned}
& m_{x} \nu_{x}\left(J_{x R}, J_{y R}\right)+m_{y} \nu_{y}\left(J_{x R}, J_{y R}\right)=l, \\
& \nu_{x}=\nu_{x 0}+\Delta \nu_{x}, \quad \nu_{y}=\nu_{y 0}+\Delta \nu_{y},
\end{aligned}
$$

where $\nu_{x 0}, \nu_{y 0}$ are the bare tunes, $\left(J_{x R}, J_{y R}\right)$ is the pair of actions which satisfy the resonance condition, and $m_{x}, m_{y}, l$ are integers. For the NX21 and NX22 experiments, these can be simplified as

$$
\begin{aligned}
& \nu_{x}\left(J_{x R}, J_{y R}\right)=21 \quad(\mathrm{NX} 21), \\
& \nu_{x}\left(J_{x R}, J_{y R}\right)=22 \quad(\mathrm{NX} 22) .
\end{aligned}
$$

The resonance width $\Delta J_{x}$, which is the full length of the resonance island, can also be calculated analytically [17]. For simplicity, only the $x$ coordinate is written hereinafter. Considering the resonance induced by $\tilde{U}_{k 0}$, the Hamiltonian $H$ can be written as

$$
H=\nu_{x 0} J_{x}+\tilde{U}_{00}\left(J_{x}\right)+\tilde{U}_{k 0}\left(J_{x}\right) \cos \left(k \psi_{x}+\chi_{k}\right) .
$$

Approximating $\tilde{U}_{00}\left(J_{x}\right)$ as

$$
\begin{aligned}
\tilde{U}_{00}\left(J_{x}\right)= & \tilde{U}_{00}\left(J_{x R}\right)+\left.\frac{\partial \tilde{U}_{00}}{\partial J_{x}}\right|_{J_{x R}}\left(J_{x}-J_{x R}\right) \\
& +\left.\frac{1}{2} \frac{\partial^{2} \tilde{U}_{00}}{\partial J_{x}^{2}}\right|_{J_{x R}}\left(J_{x}-J_{x R}\right)^{2},
\end{aligned}
$$

The resonance width can be written as

$$
\begin{gathered}
\Delta J_{x}=4 \sqrt{\frac{\tilde{U}_{k 0}\left(J_{x R}\right)}{\lambda_{x x}},} \\
\lambda_{x x}=\left.\frac{\partial^{2} \tilde{U}_{00}}{\partial J_{x}^{2}}\right|_{J_{x R}} .
\end{gathered}
$$

The space-charge-induced resonance width in the NX21 experiment is considered to be $\left(m_{x}, m_{y}\right)=(4,0)$. Then, the resonance width is

$$
\Delta J_{S C}=4 \sqrt{\frac{\tilde{U}_{40}\left(J_{x R}\right)}{\lambda_{x x}}} .
$$

The resonance width induced by the sextupoles can be obtained by calculating $\tilde{U}_{30}$ as follows [18]:

$$
\begin{gathered}
\tilde{U}_{30}=2\left|\frac{\sqrt{2}}{24} J_{x}^{3 / 2} \oint \mathrm{d} s \beta_{x}^{3 / 2} K_{2} e^{3 i \psi_{x}}\right|, \\
\Delta J_{\text {sext }}=4 \sqrt{\frac{\tilde{U}_{30}\left(J_{x R}\right)}{\lambda_{x x}}},
\end{gathered}
$$

where $K_{2}$ is the sextupole field strength. Without the spacecharge effect, $\tilde{U}_{30}$ is canceled by setting the horizontal phase $\psi_{x}$ properly in the MR. However, the space-charge effect induces shifts in $\psi_{x}$ and the resonance appears. The potentials were calculated with the shifted phase $\psi_{x}$.

\section{Calculation results}

Figure 13 shows the results of the analytical calculations with the initial conditions of the NX21 experiment. The horizontal axis represents the bare horizontal tune. For example, when the horizontal tune $\nu_{x 0}$ is 21.08 , the on-resonance action, $2 J_{x R}$, is about $9.3 \mu \mathrm{m}$, the spacecharge-induced resonance appears at 3.5-15.1 $\mu \mathrm{m}$, and the resonance driven by the sextupoles appears at $6.3-12.3 \mu \mathrm{m}$. The values for the conditions of $\nu_{x 0}=21.08,21.14,21.24$ are summarized in Table IV in order to make it easier to compare with the simulations. The space-charge-induced resonance was larger than the sextupole-induced resonance in any tune of the NX21 experiment.

The calculated values of $2 J_{x R}$ have also been plotted in Fig. 9 as black dotted lines. It shows that the analytical $2 J_{x R}$ lines are at the center of the resonance islands obtained by the simulations. 


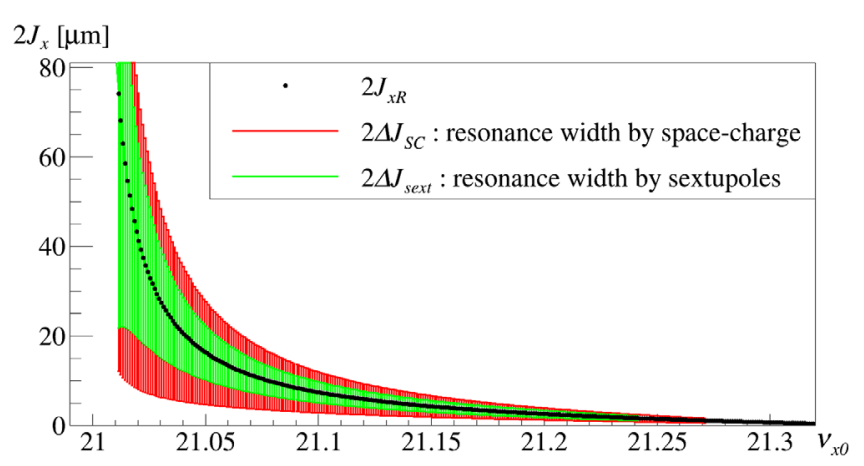

FIG. 13. The on-resonance actions $2 J_{x R}$ and the resonance widths in the NX21 experiment as a function of the bare tune obtained from the analytical calculations. The horizontal axis represents the bare horizontal tune. The black points represent the on-resonance actions $2 J_{x R}$, red lines represent the resonance widths due to the space-charge effect $2 \Delta J_{S C}$, and the green lines represent the resonance widths due to the sextupoles $2 \Delta J_{\text {sext }}$.

Figure 14 shows the results of the analytical calculations with the initial conditions of the NX22 experiment. The space-charge potential $\tilde{U}_{40}$ was canceled at $\nu_{x 0}=22 . *$ as no magnetic errors were included in the calculations. Consequently, the space-charge-induced resonances did not appear in the NX22 experiment.

The values of $2 J_{x R}$ and the resonance widths have been summarized in Table IV and the former have also been plotted in Fig. 14 as black lines. From a comparison of the analytical calculations and simulations, the analytical $2 J_{x R}$ lines are observed to be at the center of the resonance islands obtained by the simulations. The values of the resonance widths obtained from the simulations and the analytical calculations are also observed to be consistent.

In the MR, one arc section consists of eight periodic modules, and three sextupoles are located in a module. The phase advance of a module is $1.5 \pi \mathrm{rad}$. Substituting $\psi_{x}$ into Eq. (19), the sextupole potential $\tilde{U}_{30}$ is canceled. However, the shift of $\psi_{x}$ caused by the space-charge effect contributes to the resonance width. The agreement between the values of the resonance width obtained from simulations as well as analytical calculations confirms that the space-charge phase shift at the sextupoles induces a third-order resonance.

TABLE IV. The on-resonance action $2 J_{x R}$ and the resonance widths calculated analytically. The units of $2 J_{x R}, 2 \Delta J_{S C}$, and $2 \Delta J_{\text {sext }}$ are $\mu \mathrm{m}$.

\begin{tabular}{lrcc}
\hline \hline Horizontal tune $\nu_{x 0}$ & $2 J_{x R}$ & $2 \Delta J_{S C}$ & $2 \Delta J_{\text {sext }}$ \\
\hline 21.08 & 9.3 & 11.6 & 6.0 \\
21.14 & 4.2 & 4.4 & 2.7 \\
21.24 & 1.4 & 1.4 & 1.0 \\
22.05 & 12.2 & 0 & 6.9 \\
22.14 & 3.0 & 0 & 1.5 \\
22.24 & 0.7 & 0 & 0.4 \\
\hline \hline
\end{tabular}

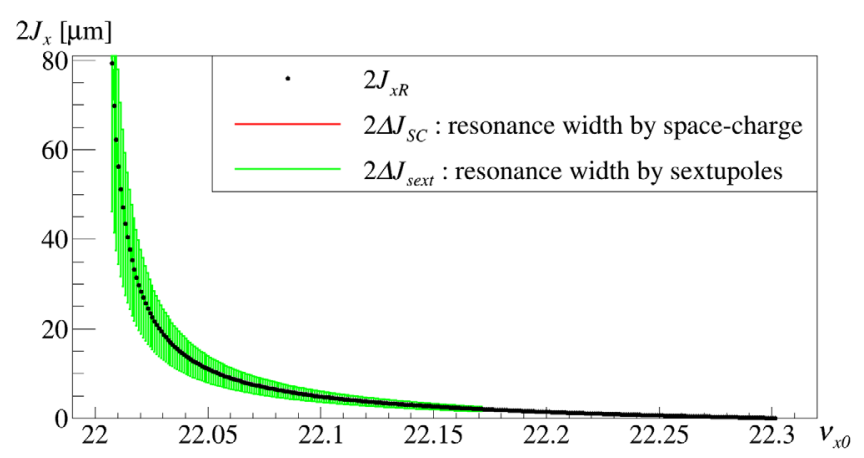

FIG. 14. The on-resonance actions $2 J_{x R}$ and the resonance widths in the NX22 experiment as a function of the bare tune obtained from the analytical calculations. The horizontal axis represents the bare horizontal tune. The black points represent the in-resonance actions $2 J_{x R}$, and the green lines represent the resonance widths due to the sextupoles $2 \Delta J_{\text {sext }}$. The resonance width due to the space-charge (red line) does not appear because it is 0 .

\section{CONCLUSIONS}

The emittance growth caused by resonances were measured in the J-PARC MR using Inj-MRPM. The targets were the space-charge-induced structure resonances at $\nu_{x}=21, \nu_{y}=21$, and the space-charge-induced nonstructure resonance at $\nu_{x}=22$.

The results were reproduced satisfactorily by SCTR simulations. The simulations indicated that the main driving source of the resonances at $\nu_{x}=21, \nu_{y}=21$ is the space-charge effect.

The resonance at $\nu_{x}=21$ affected only the horizontal emittance, while the resonance at $\nu_{y}=21$ affected only the vertical emittance.

The horizontal emittance growth, occurring due to the space-charge-induced structure resonance at $\nu_{x}=21$, measured by the profiles in 100 turns was found to be much larger than that of the space-charge-induced non-structure resonance at $\nu_{x}=22$. The emittance grew by a factor of 5 owing to $\nu_{x}=21$ and by a factor of 1.8 owing to $\nu_{x}=22$ in 100 turns.

The horizontal emittance growth depended strongly on the horizontal tunes in the NX21 experiment. In the case of $\nu_{x}=21.08$, the emittance was temporarily stable for the first 30 turns. This is because the core of the tune spread was below the integer resonance. After 30 turns, the emittance started growing owing to the core of the tune spread crossing the resonance. The growth was fast at $\nu_{x}=$ 21.14 because most of the particles were on the integer resonance. When the tune was far from the integer resonance, the growth was observed to be small.

Frozen model simulations were performed in order to evaluate the strength of the resonances. They revealed the mechanism of the emittance growth with the condition of $\nu_{x}=21 . *$. The resonance widths were also calculated analytically and compared with the corresponding results 
obtained from simulations. For the condition $\nu_{x}=21 . *$, the main cause of exciting $\nu_{x}=21$ was the space-charge effect which was confirmed by the observation of a clear fourthorder resonance appearing at $\nu_{x}=21.08$. At $\nu_{x}=22 . *$, the resonance at $\nu_{x}=22$ was induced by sextupoles. Thirdorder resonance was observed in the simulation results and is consistent with the analytical calculations.

In the future work, vertical emittance growth occurring due to the space-charge-induced resonances will be measured. Resonance evaluation by measuring the emittance growth will be applied for higher-order resonances. We are planning to introduce an optical transition radiation monitor and an MRPM which can measure several hundreds of turn-by-turn beam profiles.

The optimization of the operation tune is also ongoing for the higher beam power. The results of this work recommend the development of the operation point $\left(\nu_{x}, \nu_{y}\right)=(22 . *, 22 . *)$.

\section{ACKNOWLEDGMENTS}

We are grateful to Y. Hashimoto for setting up the Inj-MRPM and K. Sato for helping in taking the data. We would also like to thank Y. Sugiyama for providing us with the data of the wall current monitor. We thank the members of the power supply, control, monitor, rf, and injection/ extraction groups for the MR. This study was supported by the MEXT program "Program for Leading Graduate Schools" and the JSPS program "Research Fellowship for Young Scientists."

[1] T. Koseki et al., Beam commissioning and operation of the J-PARC main ring synchrotron, Prog. Theor. Exp. Phys. 2012, $02 B 004$ (2012).

[2] S. Igarashi, High-power beam operation at J-PARC, in Proc. HB'18, Daejeon, Korea, June 2018 (JACoW Publishing, Geneva, Switzerland, 2018), TUA2WD02, pp. 147-152.

[3] T. Koseki, Upgrade plan of J-PARC MR - toward 1.3 MW beam power, in Proc. IPAC'18, Vancouver, Canada, May 2018 (JACoW Publishing, Geneva, Switzerland, 2018), TUPAK005, pp. 966-969.

[4] K. Ohmi, S. Igarashi, H. Koiso, T. Koseki, and K. Oide, Study of halo formation in J-PARC MR, in Proceedings of the 22nd Particle Accelerator Conference, PAC-2007, Albuquerque, NM (IEEE, New York, 2007), THPAN040, pp. 3318-3320.
[5] F. J. Sacherer, Transverse space-charge effects in circular accelerators, Ph.D. thesis, University of California, Berkeley, 1968.

[6] S. Machida, Space-charge-induced resonances in a synchrotron, Nucl. Instrum. Methods Phys. Res., Sect. A 384, 316 (1997).

[7] S. Cousineau, S. Y. Lee, J. A. Holmes, V. Danilov, and A. Fedotov, Space charge induced resonance excitation in high intensity rings, Phys. Rev. Accel. Beams 6, 034205 (2003).

[8] Y. Hashimoto et al., Multi-ribbon profile monitor using carbon graphite foil for J-PARC, in Proc. HB'10, Morschach,

Switzerland, 2010, WEO2A01, pp. 429-433, http:// accelconf.web.cern.ch/HB2010/papers/weo2a01.pdf.

[9] M. Tanabashi et al. (Particle Data Group), Review of Particle Physics, Phys. Rev. D 98, 030001 (2018).

[10] A. Kobayashi, S. Igarashi, Y. Sato, T. Shimogawa, Y. Sugiyama, T. Toyama, and M. Yoshii, Studies on coherent multi-bunch tune shift with different bunch spacing at the J-PARC main ring, in Proc. IPAC'19, Melbourne, Australia, May 2019, MOPGW036, pp. 167-170 (JACoW Publishing, Geneva, Switzerland, 2019).

[11] http://acc-physics.kek.jp/SAD/.

[12] S. Igarashi, K. Ishii, T. Koseki, A. Molodozhentsev, K. Niki, K. Okamura, M. Tomizawa, and E. Yanaoka, Study of the J-PARC MR beam orbit based on the magnetic field measurements, in Proc. PASJ'07, Wako, Japan, August 2007, TP62, https://www.pasj.jp/web_publish/ pasj4_lam32/PASJ4-LAM32/contents/PDF/TP/TP62.pdf.

[13] T. Toyama, D. Arakawa, Y. Hashimoto, S. Lee, T. Miura, S. Muto, N. Hayashi, R. Toyokawa, and J. Kishiro, Beam diagnostics for the J-PARC main ring synchrotron, in Proceedings of the 21st Particle Accelerator Conference, Knoxville, TN, 2005 (IEEE, Piscataway, NJ, 2005), RPAT005, pp. 958-960.

[14] Y. S. Tsai, Pair production and bremsstrahlung of charged leptons, Rev. Mod. Phys. 46, 815 (1974).

[15] A. W. Chao and M. Tigner, Handbook of Accelerator Physics and Engineering, 2nd ed. (World Scientific, Singapore, 2002), p. 130.

[16] K. Ohmi and K. G. Sonnad, Beta function measurement and resonances induced by space charge force and lattice magnets, in Proc. IPAC'16, Busan, Korea, May 2016, MOPOR019, pp. 641-643 (JACoW, Geneva, Switzerland, 2016).

[17] J.L. Tennyson, The dynamics of the beam-beam interaction, in AIP Conf. Proc. No. 87, 345 (1982).

[18] S. Y. Lee, Accelerator Physics, 3rd ed. (World Scientific, Singapore, 2012). 